had a disagreeable odor from the nose for a long time. Inspection shows atrophic rhinitis. $\mathrm{Pa}$ tient has taken nasal baths at home for some time. Came to clinic in January, r89I. The vibrations in this case were followed by entire disappearance of the fotor and diminition of secretion, but the atrophy remains unchanged.

From these cases and results we may draw the following conclusions:

First-To the cleanliness which is made so essential in this mode of treatment, are due most of the speedy cures or reliefs of disagreeable symptoms, i. e., fœetor, secretion, etc.

Second. -The vibratory movements themselves may be indeed of some benefit, inasmuch as they are stimulating to the lymphatic and vascular system, but

Thirdly we must believe from the above results that the vibrations have little or no action in regenerating atrophic tissue.

In conclusion I have to express my sincere gratitude to that eminent teacher, Dr. Ottocan as well as for many other kindnesses.

London, April 22d, 189I.

\section{A NEW UNIVERSAL DOUBLE-ACTING SNARE.}

BY CHARLES A. BUCKLIN, A.M., M.D., OF NEW YORK.

The good principles of all similar instruments have been combined in this snare, and their inherent defects have been obviated.

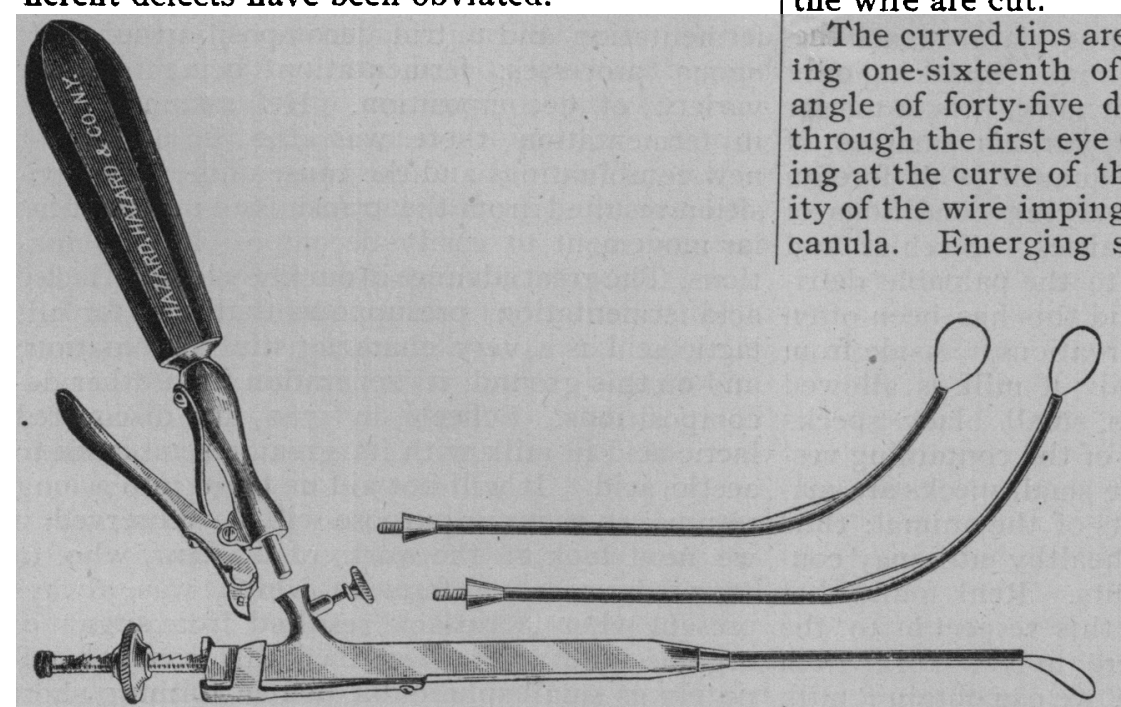
growth. transit of the curve. loop emhraces. the wire are cut. Chiari, for his permission to publish these results

motion provides a rapid-cutting one where there is no reason to expect hæmorrhage.

The handle and ratchet motion may be detached at pleasure, leaving an instrument suitable for the slow strangulation of a very vascular

The instrument has a straight tip for the nose and curved tips for reaching the larynx and naso-pharynx through the pharynx.

In curved canulas all attempts to use flexible stylets under severe traction are dangerous, as they must break sooner or later in making the

With this wire écraseur every benign neoplasnr or hypertrophy found in the nose, naso-pharynx or pharynx can be removed at a single sitting in a manner which commands the approval of the most conservative operator.

This instrument also fully meets all the requirements met by Stoerks' laryngeal guillotine or wire écraseur, and furnishes one not provided with laryngeal forceps with a simple and effective means of extracting many foreign bodies which lodge in and about the larynx.

If the instrument is firmly secured at the detaching joint, the wire properly fastened, and the clearances of the ratchet under-strain are $\frac{1}{3} 2$ of an inch, it will never fail to cut any growth the

The straight canula is armed with wire by passing it through the eyes of the slightly projecting stylet from opposite sides. The required loop having been formed, the stylet is drawn by the ratchet motion, and the projecting ends of

The curved tips are armed with wire by bending one-sixteenth of an inch of the end at an angle of forty-five degrees. It is then passed through the first eye of the stylet into the opening at the curve of the canula, with the convexity of the wire impinging on the concavity of the canula. Emerging sufficiently to form a loop, it is returned through the curved canula, with its convexity impinging on the concave surface of the canula. When the wire appears at the opening, it is twisted until the bent point is opposite the second eye in the stylet, which it will enter easily, and the loop is complete.

By the pressure of the thumb on the tip of the The wire is attached to a solid stylet, the ob- instrument, the wire loop, after having been jections to which are overcome by the powerful drawn, can be returned instantly to position for ratchet motion which draws it. A screw motion further operation through any of the tips.

is also attached to the same stylet, thus enabling For further particulars and history of steel the operator to use a very slow-cutting snare, wire snares, see New York Medical Record, July where bæmorrhage is feared, while the ratchet 4,1891 . 